\title{
Pursuing a Possibility of Fabricating Moisture Measurement Devices Using Microwaves Passing through Materials -Proposition of Measuring Quantity of Water Level Using Microwaves Passing through the Water Tank as the First Step of Development-
}

\author{
Toshinari Akimoto, ${ }^{1 *}$ Keisuke Maruyama, ${ }^{2}$ Toyomi Miyagawa, ${ }^{1}$ \\ Yuichi Nakazato, ${ }^{1}$ and Yasuhiro Kushihashi ${ }^{1}$ \\ ${ }^{1}$ Department of Robotics, Nippon Institute of Technology, \\ 4-1 Gakuendai, Miyashiro, Minami-Saitama, Saitama 345-8501, Japan \\ ${ }^{2}$ Graduate School of Mechanical Systems Engineering Major, Nippon Institute of Technology, \\ 4-1 Gakuendai, Miyashiro, Minami-Saitama, Saitama 345-8501, Japan
}

(Received March 13, 2019; accepted September 10, 2019)

Keywords: microwaves, moisture measurement, received signal strength, propagation delay time

In recent years, an increasing number of sensors are being required in various industrial or automated production techniques. In particular, the need for moisture measurement is high, but a noncontact high-precision moisture sensor has not been put to practical use. Therefore, in this research, we focused on a moisture sensor that transmits microwaves and tested two methods for evaluation and verification. In the experiment, the water in the water tank was measured using radio waves of $2.4 \mathrm{GHz}$. As a result, although measurement could not be performed with high accuracy using the received signal strength, measurement using the propagation delay time was highly accurate. This result will provide an index for the application of moisture sensors using microwaves in the future.

\section{Introduction}

In industrial production, the measurement of the moisture condition is one of the most important technologies. For example, when using wood in industrial production, it is necessary to measure the moisture content of the wood. Wood varies in size depending on the condition of moisture. ${ }^{(1)}$ In manufacturing, it is important to measure the moisture condition to improve size accuracy. Also, the manufacture of high-performance concrete requires the measurement of the moisture state during the concrete manufacturing process. ${ }^{(2,3)}$ In addition, to diagnose the deterioration of concrete structures, it is also necessary to measure the water condition in concrete. For the moisture measurement of concrete, a dielectric hygrometer or the like, which can detect changes in capacitance, is used. ${ }^{(4,5)}$ However, this is a measurement of the moisture condition of only the surface part. 
Furthermore, in industrial production, it is important to measure the water condition in order to maintain the quality of food, which has become of prominent importance in recent years. Similarly, various sensors have been developed for automation in agricultural industrialization. ${ }^{(6)}$ In the automatic production of agricultural products, it is considered very important to measure the moisture status of crops and soil, and conventionally, various systems for measuring soil moisture have been developed. ${ }^{(7-12)}$

As described above, the technology for measuring the water condition has become one of the very important issues as industrialization and automation spread in various fields.

When applying moisture measurement to industrial production, considering its convenience and applicability, important elements are as follows:

- The sensor does not need physical contact with the material.

- The sensor is fast enough for online measurement.

If the sensor is in contact with an object, the measurement results depend on the condition of the contact surface. There is a risk of damage if sensors come into contact. Furthermore, the measurement time of sensors also affects the number of products manufactured, given their use on the production line. Hence, we constructed a moisture meter that uses microwaves and performed evaluation experiments. By using microwaves, it is possible to realize noncontact measurement in real time.

Many of the measuring instruments using microwaves use the reflection of microwaves. However, in such methods, the surface is measured and it is difficult to estimate the internal state of the object.

Microwaves are easily absorbed by water, but they cannot pass through so much water. For this reason, it has been considered that moisture measurement using microwaves passing through materials is difficult.

However, moisture measurement is not impossible. If the amount of water that can be measured by the microwaves passing through is clarified, it can be applied to many measurement systems.

Therefore, in this research, we developed a moisture measurement system using microwaves that propagate through materials for the purpose of measuring the inside of an object without contact.

In this study, for basic experiments, we developed two measurement methods, one using the change in received signal strength and the other using the propagation delay time. We report each evaluation experiment.

\section{Materials and Methods}

There are two major parameters that can be measured by transmitting radio waves, namely, the received signal strength and radio wave propagation time. In this study, measurement experiments were conducted for each parameter and comparative evaluation was performed. In addition, in the basic study experiments, we passed radio waves through water in a water tank to facilitate comparison with theoretical values. The amount of water in the water tank was changed to change the amount of water through which radio waves permeate. Each experimental method is described below. 


\subsection{Measurement of received signal strength}

When radio waves pass through water, they are attenuated by scattering and absorption. We measured the changes in received signal intensity owing to this attenuation and created a system for estimating the water content.

Figure 1 shows the experimental setup used in this study. The water tank was placed between the transmitting and receiving antennas that transmit and receive radio waves, respectively, and the received signal strength for each water level that varied in steps of $1 \mathrm{~mm}$ in the water tank was measured ten times in the magnetic shielding chamber and averaged. The conditions of the transmission wave are shown in Table 1.

As a microwave transmitter, CC2543-CC2544DK from Texas Instruments was used. In this development kit, wireless communication can be evaluated by using the attached $2.4 \mathrm{GHz}$ radio module. It is possible to switch the transmission power and modulation scheme, and to conduct experiments under various conditions. We used this module as a transmitter to output an unmodulated continuous wave.

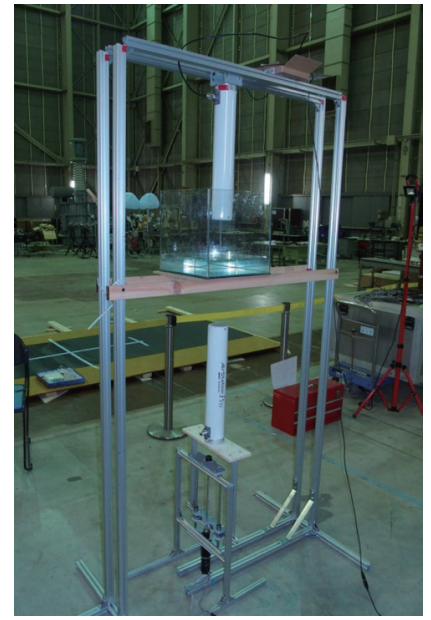

(a)

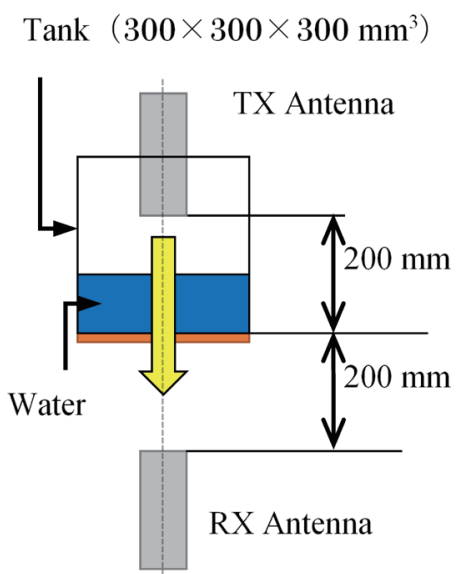

(b)

Fig. 1. (Color online) (a) Photograph of experimental setup used in this study. (b) Scheme of the setup.

Table 1

Antenna specifications and transmission conditions.

\begin{tabular}{lc}
\hline TX antenna, RX antenna & \\
\hline Voltage standing wave ratio $(\leq)$ & 1.5 \\
Gain $(\mathrm{dBi})$ & 14 \\
Half-power beamwidth $\left(^{\circ}\right)$ & $32 \pm 5$ \\
\hline RX antenna A, B & \\
\hline Voltage standing wave ratio $(\leq)$ & 2 \\
Gain $(\mathrm{dBi})$ & 10 \\
Half-power beamwidth $\left(^{\circ}\right)$ & 50 \\
\hline TX frequency $(\mathrm{Hz})$ & $2.479 \times 10^{9}$ \\
\hline TX power $(\mathrm{dBm})$ & 4 \\
\hline Modulation format & Unmodulated \\
\hline
\end{tabular}


On the receiving side, the small RF Explorer-3G Combo spectrum analyzer from Seeed Studio was used to measure the received signal strength $(\mathrm{dBm})$ of the radio waves.

The antenna of the transmitter was connected to WLE-HG-DYG, a directional antenna from BUFFALO, with the aim of concentrating the transmitted radio waves and irradiating the object to be measured. The same directional antenna was also connected to the antenna on the receiving side.

\subsection{Measurement of propagation delay time using an oscilloscope}

The radio waves propagate at the speed of light in vacuum, but they decelerate in the medium and the propagation time is delayed. Using this property, we will measure the water content by comparing the propagation times with the reception point of radio waves transmitted through water and air.

Therefore, in this experiment, as shown in Fig. 2, receiving antenna B was installed so that it mainly received radio waves transmitted through water, and receiving antenna A mainly received radio waves transmitted through air.

Then, water was poured into the water tank, and the waveforms received by each antenna at each water level that varied in steps of $1 \mathrm{~mm}$ were recorded using an oscilloscope (TDS7404B Tektronix), and the propagation delay time at antenna B was determined from the waveform (Fig. 3). Table 1 shows the characteristics of antennas and the conditions of the transmitted wave. The transmission frequency was set to the same value as that in the received signal strength experiment. Since the distance between antennas also affects the propagation time of radio waves, the propagation delay time at the water level of $0(\mathrm{~mm})$ is omitted because it is measured as the propagation delay time due to the distance between antennas.

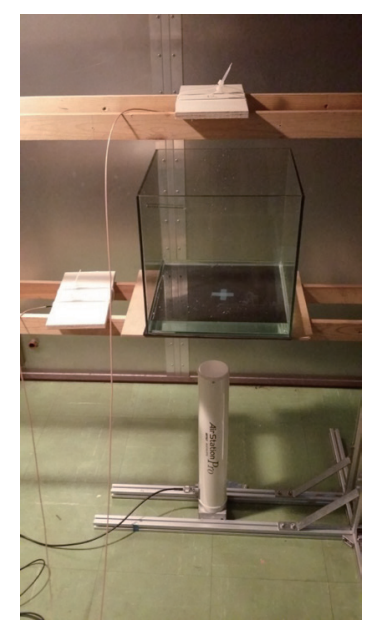

(a)

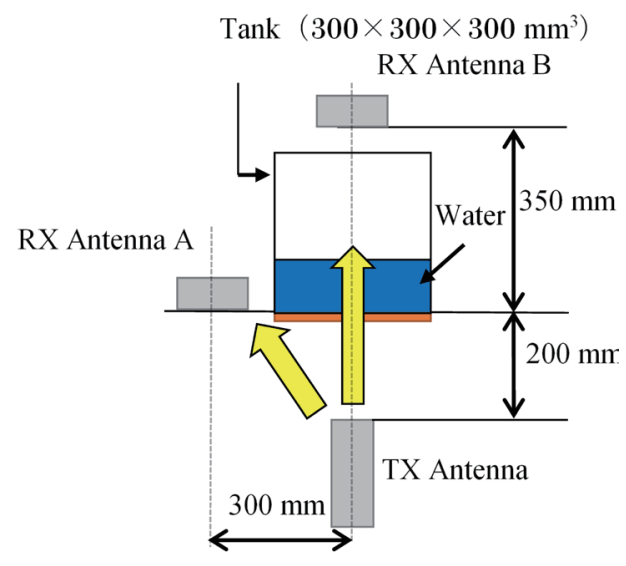

(b)

Fig. 2. (Color online) (a) Photograph of experimental setup used in this study. (b) Scheme of the setup. 


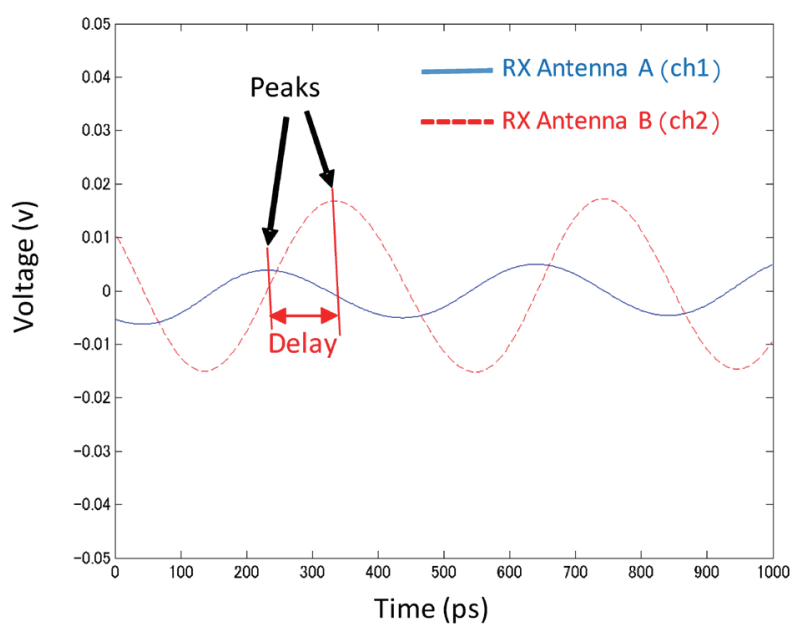

Fig. 3. (Color online) Sample results of measurement with an oscilloscope (water level of $5 \mathrm{~mm}$ ).

\section{Results}

The relationship between the water level and the attenuation of the received signal strength was calculated as a theoretical value and compared with the measured value. Equations (1) and (2) were used to calculate the theoretical value.

$$
\begin{gathered}
D=\frac{3.31 \times 10^{10}}{f \sqrt{\varepsilon_{r}} \tan \delta} \\
\alpha=\frac{A}{8.7 \times 10^{-3} D}
\end{gathered}
$$

In Eq. (1), ${ }^{(13)} D$ is the half-power depth (mm), $f(\mathrm{~Hz})$ is the frequency of radio waves, $\varepsilon_{\gamma}$ is the relative permittivity, and $\tan \delta$ is the dielectric loss angle. When the frequency $f$ is $2.479 \times 10^{9}(\mathrm{~Hz})$, the constant $\varepsilon_{r}$ of water is 76.7, and when $\tan \delta$ is 0.16 , the half-power depth is about $9.5 \mathrm{~mm}$.

In Eq. (2), $\alpha$ is the damping constant $(\mathrm{Np} / \mathrm{mm})$ and $A$ is the amount of attenuation $(\mathrm{dB})$. Substitute $D$ obtained using Eq. (1) into Eq. (2). Then, since $D$ is the half-power depth, $3 \mathrm{~dB}(\approx 0.5)$ is substituted for $A$. $\alpha$ was calculated to be about $36.2 \mathrm{~Np} / \mathrm{mm}$. This $\alpha$ was substituted into Eq. (3), which is transformed from Eq. (2), and the relationships between the water level $D(\mathrm{~mm})$ and $A$ plotted in Fig. 4 for the theoretical and measured values were compared.

$$
A=8.7 \times 10^{-3} \alpha D
$$

The same tendency was confirmed in both the measured and theoretical values. However, it was confirmed that the measured value was far from the theoretical value depending on the water level. 
Next, the relationship between the water level and the propagation delay time was calculated using Eq. (4) and compared with the measured value.

$$
\varphi=\frac{2 \pi f D \sqrt{\varepsilon_{r}}}{c}
$$

Equation (4) represents the delay phase $\varphi(\mathrm{rad})$ that occurs until a radio wave of frequency $f$ $(\mathrm{Hz})$ travels through a medium with the relative permittivity $\varepsilon_{r}$ and depth $D(\mathrm{~mm})$ and reaches the reception point. $c$ is the speed of light $\left(3 \times 10^{11} \mathrm{~mm} / \mathrm{s}\right)$.

Then, assuming that $f$ is $2.479 \times 10^{9} \mathrm{~Hz}$ and $\varepsilon_{r}$, which is a relative permittivity of water, is 76.7, the relationship between the water level $D(\mathrm{~mm})$ and $t$ is obtained using Eq. (5), which shows the relationship between $\varphi$ and the propagation delay time $t$ (s). Subsequently, Eq. (6) is derived. The measured propagation delay times and the theoretical values calculated using Eq. (6) are plotted in Fig. 5.

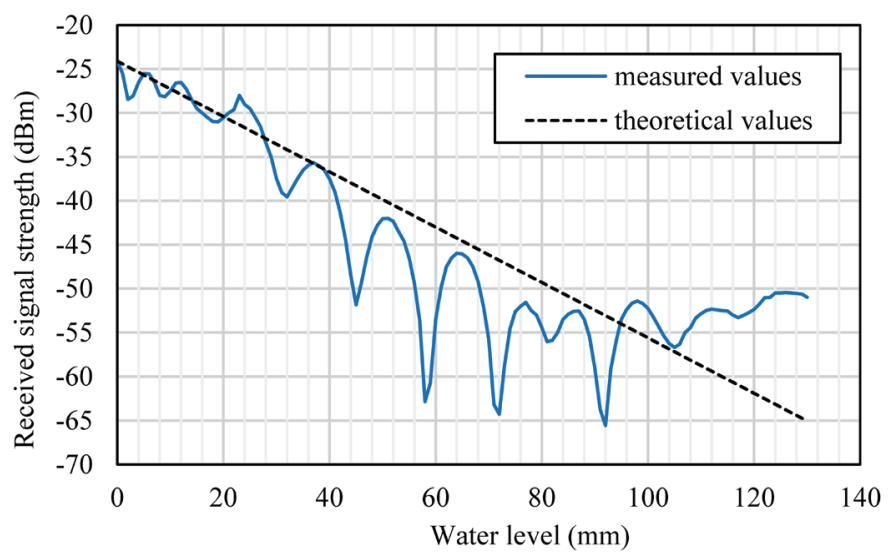

Fig. 4. (Color online) Theoretical and measured received signal strengths.

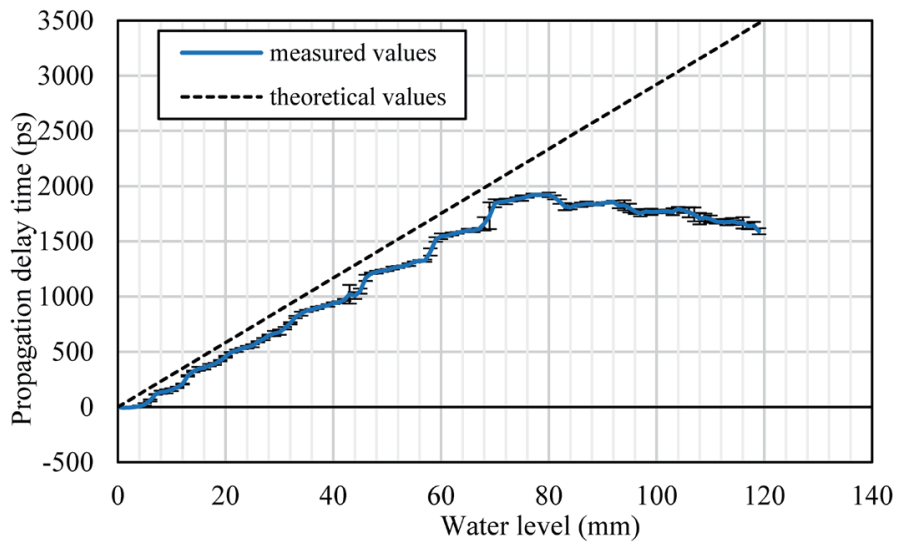

Fig. 5. (Color online) Theoretical and measured propagation delay times. 


$$
\begin{gathered}
t=\frac{\varphi}{2 \pi f} \\
t=\frac{D \sqrt{\varepsilon_{r}}}{c}
\end{gathered}
$$

The experimental results are very close to the theoretical values. However, when the water level exceeds $80 \mathrm{~mm}$, they are far from the theoretical values.

\section{Discussion}

As a result of the experiment, we found that the same experimental results as the theoretical values were obtained for the received signal strength and propagation delay time by transmitting radio waves through water. However, the measured received signal strength largely deviated from the theoretical value after a certain point. It is considered that this deviation is caused by a phenomenon called fading, which is due to the diffraction and scattering of radio waves. Strong and weak received signals occur owing to the resonance of radio waves along multiple paths. The same result is obtained when the experiment is repeated several times. From this, it is considered that the effect of fading might be reduced by enhancing the directivity of the antenna and limiting the path of radio waves.

However, overall, roughly the same result as the theoretical value can be obtained, so it may be possible to use only the received signal strength depending on the application. Moreover, in the measurement result of the propagation delay time, it was possible to obtain a result very close to the theoretical value. Since the change in propagation delay time is also linear up to the water level of about $80 \mathrm{~mm}$, it is considered that calibration based on the experimental results can realize accurate measurement of water content.

Although the measurement result largely deviates from the theoretical value beyond the water level of $80 \mathrm{~mm}$, it is considered that the radio wave is greatly attenuated and that the transmitted radio wave cannot be measured.

These findings confirmed that high-precision measurement can be realized by using the propagation delay time to determine the water content. Also, it is considered that more reliable results can be obtained by measuring the received signal strength and propagation delay time together.

The measurement accuracy of the propagation delay time is higher than that of the received signal strength because the delay time is less susceptible to fading. However, the measurement requires an accuracy of several picoseconds.

When applying the developed system to the measurement of moisture in a material, it is necessary to consider the effects of the moisture distribution and the dielectric constant of the material itself. However, even if it is $100 \%$ water, it could be confirmed that the measurement was possible if it was up to about $80 \mathrm{~mm}$. 


\section{Conclusions}

In this research, we conducted an experiment to measure two parameters that can be measured by transmitting radio waves, namely, the received signal strength and radio wave propagation time.

The results of the experiment were as follows.

- In the measurement of the received signal strength, it was difficult to measure small changes in water volume because of the effect of fading.

- In the measurement of the propagation delay time, the effect of fading was small, and the change in water volume could be measured with high accuracy.

- The measurement using $2.4 \mathrm{GHz}$ radio waves was difficult when the amount of water exceeded $80 \mathrm{~mm}$.

Sensors using microwaves can realize sufficiently fast noncontact measurements. The state within an object can also be measured by using this infiltration system method. On the basis of these findings, it is expected that moisture measurement using microwaves will be applied in various fields in the future.

The results of this study demonstrate the accuracy and limitations needed to develop a sensor capable of measurements with microwaves. In the future, it is expected that different moisture content measurements will be made using microwaves. At that time, we believe that this research result will be an indicator of moisture sensor development using radio wave transmission.

\section{Acknowledgments}

This work was partially supported by JSPS KAKENHI Grant Numbers 18K12113.

\section{References}

1 R. Mvondo, P. Meukam, J. Jeong, D. Meneses, and E. Nkeng: Results Phys. 7 (2017) 2096. https://doi. org/10.1016/j.rinp.2017.06.025

2 K. Wang and J. Hu: J. Adv. Concr. Technol. 3 (2005) 371. https://doi.org/10.3151/jact.3.371

3 S. Rubene, M. Vilnitis, and J. Noviks: Procedia Eng. 123 (2015) 471. https://doi.org/10.1016/j.proeng.2015.10.096

4 N. Yuasa, Y. Kasai, and I. Matsui: J. Struct. Constr. Eng. (Trans. AIJ) 62 (1997) 13 (in Japanese). https://doi. org/10.3130/aijs.62.13_1

5 M. Andrzej and M. Marta: Procedia Eng. 153 (2016) 483. https://doi.org/10.1016/j.proeng.2016.08.162

6 A. Zujevs, V. Osadcuks, and P. Ahrendt: Procedia Comput. Sci. 77 (2015) 227. https://doi.org/10.1016/ j.procs.2015.12.378

7 B. Ortuani, A. Benedetto, M. Giudici, M. Mele, and F. Tosti: Procedia Environ. Sci. 19 (2013) 446. https://doi. org/10.1016/j.proenv.2013.06.051

8 S. U. S. Lekshmi, D. N. Singh, and M. S. Baghini: Measurement 54 (2014) 92. https://doi.org/10.1016/ j.measurement.2014.04.007

9 B. Marco: HortTechnology 21 (2019) 293. https://doi.org/10.21273/HORTTECH.21.3.293

10 K. Foughali, K. Fathallah, and A. Frihida: Procedia Comput. Sci. 130 (2018) 575. https://doi.org/10.1016/ j.procs.2018.04.106

11 I. Mohanraj, K. Ashokumar, and J. Naren: Procedia Comput. Sci. 93 (2016) 931. https://doi.org/10.1016/ j.procs.2016.07.275

12 Y. Huang, Z. Chen, T. Yu, X. Huang, and X. Gu: J. Integr. Agric. 17 (2018) 1915. https://doi.org/10.1016/S20953119(17)61859-8

13 A. R. Von Hippel: Dielectric Materials and Applications (MIT Press, Boston, 1954) p. 438. 